\title{
Reversal of Novel Anticoagulants: Role of Clotting Factors and Complex Concentrates
}

\author{
A. Josh Roberts • Mark E. Sutter • William E. Dager
}

Published online: 27 September 2013

(C) Springer Science+Business Media New York 2013

\begin{abstract}
Dabigatran, rivaroxaban, and apixaban are effective second-generation oral anticoagulants with an acceptable bleeding risk profile when compared to warfarin. Unlike warfarin, these agents do not have an antidote to reverse their anticoagulant effects should a major bleeding event occur. Prothrombin complex concentrates and activated recombinant factor VII have been investigated as potential reversal agents in animal bleeding models and to monitor the in vitro and ex vivo response of laboratory parameters in the setting of these new oral anticoagulants. These potent, non-specific pro-hemostatic agents have been used in case reports with varying degrees of success. The best management approach in the setting of an active bleeding event remains unclear at this time. The purpose of this review is to discuss the current available data and management considerations and ultimately aid the clinician in the use of clotting factors and complex concentrates in the setting of a pathologic bleeding event.
\end{abstract}

\footnotetext{
A. Josh Roberts $(\varangle)$. W. E. Dager

Department of Pharmacy, University of California,

Davis Medical Center, 2315 Stockton Blvd, Sacramento,

CA 95817, USA

e-mail: aaron.roberts@ucdmc.ucdavis.edu

W. E. Dager

e-mail: william.dager@ucdmc.ucdavis.edu

A. Josh Roberts · W. E. Dager

University of California, San Francisco School of Pharmacy,

San Francisco, CA, USA
}

\author{
A. Josh Roberts - W. E. Dager \\ Department of Internal Medicine, University of California, \\ Davis School of Medicine, 4150 V Street, PSSB 3100, \\ Sacramento, CA 95817, USA
}

Keywords Dabigatran - Rivaroxaban - Apixaban · Prothrombin complex concentrate $\cdot$ Recombinant factor VII activated · Factor eight inhibitor bypassing activity

\section{Introduction}

The landscape of available oral anticoagulants has changed dramatically over the past several years with the FDA approval of the direct thrombin inhibitor, dabigatran, and direct factor Xa inhibitors, rivaroxaban and apixaban. Largescale clinical trials have shown these second-generation oral agents to be effective anticoagulants with an acceptable bleeding risk profile when compared to warfarin [1-3]. In the case of a required non-urgent procedure or need for surgery, it may suffice to withhold the oral anticoagulant for approximately two half-lives for minor procedures and at least five half-lives for major procedures. This strategy has

\author{
M. E. Sutter \\ Division of Medical Toxicology, University of California, Davis \\ School of Medicine, Sacramento, CA, USA \\ e-mail: mark.sutter@ucdmc.ucdavis.edu \\ M. E. Sutter \\ Department of Emergency Medicine, University of California, \\ Davis Medical Center, 4150V Street, PSSB 2100, Sacramento, \\ CA 95817, USA \\ W. E. Dager \\ Touro School of Pharmacy, Vallejo, CA, USA
}


resulted in similar peri-procedural bleeding in the case of dabigatran when compared to warfarin [4].

It remains unclear at this time, however, what the clinician is to do when the patient is in need of an emergent procedure or is experiencing a major bleeding event in the presence of a second-generation oral anticoagulant. Specific antidotes are currently in varying phases of development, leaving the clinician to consider non-specific prohemostatic agents such as activated recombinant factor VII (rFVIIa), three-factor prothrombin complex concentrate (PCC3), four-factor prothrombin complex concentrate (PCC4), or activated prothrombin complex concentrate (aPCC, factor eight inhibitor bypassing activity) in attempts to independently overcome the effects of the anticoagulant [5-7]. Unfortunately, few data are available to guide the practitioner in how these pro-hemostatic agents should be used.

It seems intuitive to reverse any degree of anticoagulation in the presence of a major bleed or prior to an emergent invasive procedure. It remains however unclear in certain situations whether the administration of a reversal agent positively alters long-term outcomes [8]. It also remains unclear whether the administration of a pro-hemostatic agent in the presence of a second-generation oral anticoagulant places a patient at risk for thromboembolic events, as has been noted with their use in the reversal of warfarin [9-11]. There are no guidelines or consensus statements describing which pro-hemostatic agents to use in the setting of a major bleeding event due to a secondgeneration anticoagulant.

Much of the current literature discussing reversal of the new anticoagulants is confined to in vitro analyses and animal models [12]. There are currently only human case reports describing the use of non-specific pro-hemostatic agents in the setting of a major bleeding event or prior to a planned procedure [13-15]. In all, this leaves the clinician to sift and sort through in vitro laboratory studies and case reports to determine when and how to use non-specific prohemostatic agents. The scope of this review is to explore the role of clotting factors and pro-thrombin complex concentrates in the emergent reversal of the new oral anticoagulants' effects for the practicing clinician.

\section{Prior to a Bleeding Event}

Development and implementation of a clinical decision support system for the treatment of a patient experiencing a major bleeding event while on a new oral anticoagulant prior to a patient presenting with major bleeding event could be critical for positive outcomes. The medical institution should develop guidelines in advance of a major bleeding event that clearly outline the process for treatment to reduce potential delays in life-saving therapies. Relevant services (i.e. surgery, nephrology, hematology, and institutional anticoagulation specialists) need to be involved in guideline development to address and resolve any potential barriers to their involvement and intervention. In the development of the process, key decision makers should account for available institutional resources (e.g., urgent/ emergent dialysis), medical and pharmacy informatics (e.g., medication ordering and profiling), and available formulary reversal agents along with dosing strategies. The guidelines should identify whom to contact in case of a major bleeding event and the responsibilities of each provider and department. Overall, this document should be clear and streamlined in an effort to minimize treatment delays.

Staff involved in the care of these possibly critically ill patients should receive periodic in-servicing on the process. Staff should know how to access necessary materials to care for the patient. They should also receive professionappropriate drug information and dosing ranges, along with proper admixing relevant to the product being used.

The goals of reversal need to be clearly identified and delineated. Patient-specific factors and the desired degree of reversal should be taken into account rather than blindly administering a patented dose of a specific reversal agent. The administration of a 'one size fits all' dose may not be desirable in all situations. In certain instances where an urgent procedure is necessary where the goal is to prevent a bleeding event from occurring, it may be prudent to administer a smaller dose of a pro-hemostatic agent to lower the degree of anticoagulation rather than fully override the anticoagulant's effects. In cases of bleeding where time allows, the relatively short half-life and the rate of administration of certain reversal agents may allow for titration to effect; this should be the ideal as the use of nonspecific pro-hemostatic agents places the patient at significant risk of an arterial and venous thromboembolic event in both the short and long term.

\section{Emergency Care of Bleeding Events}

\section{Initial Identification}

In treating patients with a suspected or confirmed bleeding event, the potential lifesaving therapy may depend on the anticoagulant influencing the bleed. It is important to obtain an accurate medication history when possible, including time of last dose and indication of treatment. If this information is unobtainable, one must combine laboratory evaluation and clinical assessment to provide optimal patient care. The entire clinical presentation should be addressed instead of single observations or laboratory values. 
Laboratory Evaluation

One of the major advantages to these newer agents is the lack of need for routine therapeutic drug monitoring. While this is extremely advantageous to the patient for day-to-day life, it often proves to be a difficult task to care for the bleeding patient. Initial routine laboratory tests should include a complete blood count, chemistry panel, international normalized ratio (INR), and activated partial thromboplastin time (aPTT). Elevated coagulation values could signal the presence of an anticoagulant, and further tests may be warranted. One of the major issues with these newer anticoagulation medications is the inability of traditional laboratories to quantify the degree of anticoagulation. At certain institutions, quantifiable levels of secondgeneration oral agents may be available and thus could be helpful laboratory tests to include in the initial panel to help in the overall medical management. Laboratory tests are becoming available to quantify the effects of these agents utilizing the diluted thrombin time or ecarin clotting time (ECT); however, these tests may not be clinically available in a timely fashion. Physicians will need to adapt to limitations in availability in laboratory procedures in the care of patients with these newer agents. Further research into the quantification of the degree of anticoagulation is warranted since these agents are becoming more prevalent.

\section{Clinical Assessment}

Despite these new medications, physicians must base initial care on the clinical stability of the bleeding patient. The simple dichotomous evaluation of stable versus hemodynamically unstable patients is the most important decision. This grouping of patients often dictates not only choices of resuscitation, but also dosages of medications and products needed.

Resuscitation of the unstable patient starts with largebore intravenous access and the initiation of volume. With active bleeding, early initiation of packed red blood cells (PRBCs) is often prudent given the scenario. Additionally, the use of fresh frozen plasma (FFP) is also typically warranted as both provide volume with colloid oncotic pressure to optimize perfusion of vital organs. However, clinical experience to date has shown that FFP alone likely will not overcome the anticoagulant's effects and additional considerations are needed.

Unlike warfarin where specific guidelines of reversal exist, no comprehensive studies to date provide us with optimal reversal guidelines for either the oral direct thrombin or direct Xa inhibitors. Until these studies have been completed, it is reasonable to choose your reversal strategy based on existing literature and the pharmacologic mechanism of action. For the factor Xa inhibitors, the use of PCC3, PCC4, or factor eight inhibitor bypassing activity
(FEIBA) would provide a reasonable starting point in addition to your standard of care as mentioned earlier. As no head-to-head comparative studies have been done, agent selection may likely depend on the institutional formulary and product availability. If faced with an unstable patient with bleeding that is believed to be due to a direct factor IIa inhibitor that has activity lower down the common pathway of the clotting cascade, the chance for effective reversal is even more limited. Experiences to date regarding the use of rFVIIa have not been as encouraging [16].

There is insufficient experience with reversal in actively bleeding patients receiving a second-generation anticoagulant. Most investigations, in vitro and ex vivo, have evaluated rFVIIa, PCC4, and FEIBA, but not PCC3. It is unclear if any meaningful clinical differences exist between the various PCC products. A successful case report does exist using FEIBA to reverse a bleeding complication secondary to dabigatran during a cardiac ablation procedure [14]. Of note, dabigatran is amenable to hemodialysis and can be considered depending upon risk/benefit analysis, including the placement of a dialysis catheter [17]. If patients are bleeding but are hemodynamically stable, it is important to gather as much historical data as possible.

Specifically, time since last dose, any missed or excessive dose, and information regarding hepatic or renal dysfunction are important factors on choosing optimal therapy. Depending on the above information, observation without pharmacological intervention may be appropriate depending on the clinical scenario. If the bleeding requires pharmacologic intervention, PCCs and FEIBA, as mentioned above, could be first-line agents. FFP or rFVIIa probably plays a limited role in this setting and would only be used if the patient becomes unstable or the above agents are not effective. Further information on each of the specific agents is discussed below.

\section{Special Situations Requiring Urgent and Emergent} Procedures

Historically, FFP has been the mainstay of therapy for decades for the hemodynamically unstable patient due to its composition including clotting factors and volume. Another option for a patient in need of an urgent or emergent procedure includes the use of a small dose of a pro-hemostatic agent immediately preceding the event. The intravenous administration of $8 \mathrm{U} / \mathrm{kg}$ of FEIBA immediately prior to dialysis catheter placement has been used successfully in maintaining hemostasis despite supratherapeutic levels of dabigatran [13]. The timing of the prohemostatic agent administration may be important. A previous analysis by the FFP administration in the setting of warfarin showed that the effects on the INR dwindled over time [18]. These findings are important as they suggest it may be feasible to place a dialysis catheter or preform an 
urgent/emergent procedure in the presence of dabigatran immediately post-administration of FEIBA when the activated clotting factors are at their peak concentration. The effects of this potent pro-hemostatic agent may wane, however, as demonstrated with FFP, over time. While doses greater than $8 \mathrm{U} / \mathrm{kg}$ may provide equal hemostatic effects, the potential for thromboembolic complications may increase as dabigatran is removed from circulation. This concept likely holds true for any clotting factor concentrate administered for hemostasis prior to a procedure.

\section{Bleeding Event}

When faced with a patient who is known to be on a new oral anticoagulant, physicians must understand that traditional laboratory markers might not adequately reflect the degree of anticoagulation. Depending on the assay, the secondgeneration anticoagulant agents can cause alterations in the reported results (Table 1). The degree may depend on other factors influencing the assay and the amount of anticoagulant present. As always, clinical history and hemodynamic stability serve as guides for optimal medical management. Thorough evaluation of the patient's medical history to evaluate additional factors such as concurrent uremia or anti-platelet agents also factor in treatment strategies. Specifically, platelet transfusion and use of DDAVP can be considered if platelet function is not optional in addition to dealing with the new anticoagulants. If hypovolemia is a concern, transfusions with PRBC and FFP are still the initial mainstays of treatment. However, these traditional therapies are likely not going to be adequate therapy alone for newer anticoagulants. Additionally, if immediate volume is not required, physicians have multiple options to consider, and these are discussed in detail below.

- What should the ER clinician do when presented with a bleeding patient?

- Initial assessment including a thorough medical history

- Laboratory tests (Table 1)

Table 1 Coagulation assessment laboratory testing

\begin{tabular}{lll}
\hline Agent & Test & Comment \\
\hline Dabigatran & Chromogenic ECT, dilute TT, & Sensitivity: \\
& TT, aPTT, INR & aPTT > PT/INR \\
Rivaroxaban/ & Chromogenic anti-Xa*, PT/ & Sensitivity: PT/ \\
apixaban & INR*, aPTT & INR $>$ aPTT \\
\hline
\end{tabular}

aPTT activated partial thromboplastin time, ECT ecarin clotting time, $I N R$ international normalized ratio, $P T$ prothrombin time, $T T$ thrombin time

* Assays should be calibrated to the agent being measured
- Caution should be exercised as some hemostatic agents may normalize coagulation laboratory values while still in the presence of therapeutic concentrations of the new oral anticoagulants

- Watch for possible re-bleeding if a prohemostatic agent is given as clinical effects may wear off before anticoagulation effects have resolved

- Imaging if necessary

- Notify institutionally recognized experts in anticoagulation/hemostasis

- Assess if patient should be transferred to an institution that has the necessary interventions. Communicate in advance to limit management delays.

- When should the clinician consider reversing anticoagulation effects? (Table 2)

- Major bleeding event

- Titrate the dose of the pro-hemostatic agent by administering small, frequent doses until clinical effect if time permits

- Administrating pro-hemostatic agent should not be considered a "one and done"

- Depending on the historical scenario and concomitant medical problems such as renal failure, patients may require additional dosing of pro-hemostatic agents as their effect wears off

- Dabigatran: consider emergent hemodialysis

- Important to have nurses and pharmacists available for drug information and facilitation of blood product and medication acquisition and administration

Table 2 Strategies for establishing hemostasis

\begin{tabular}{ll}
\hline Agent & Pro-hemostatic agents \\
\hline Dabigatran & aPCC $*, \uparrow$, PCC4, PCC3, rFVIIa** \\
Rivaroxaban & aPCC, PCC3, PCC4, rFVIIa** \\
Apixaban & aPCC, PCC3, PCC4, rFVIIa** \\
\hline
\end{tabular}

* $25 \mathrm{U} / \mathrm{kg}$ to start can be considered in urgent situations

$\dagger$ Lower doses $(8 \mathrm{U} / \mathrm{kg}$ ) have been used immediately prior to invasive procedures such as line insertions

** rFVIIa: data supporting one agent over another are unclear. Current in vitro/ex vivo observations are not as encouraging 
- Urgent/emergent invasive procedures

- Consider using smaller doses of pro-hemostatic agents than those used in an imminent lifethreatening bleeding event

\section{Pro-hemostatic Agents}

\section{Fresh Frozen Plasma}

FFP is a pooled human blood product commonly used in the reversal of anticoagulation due to warfarin or when there is a depletion of clotting factors. FFP alone is not thought to be an effective reversal agent because of bleeding in the setting of dabigatran [19], nor are there any published data to date on its sole use in reversing activated factor X inhibitors. While FFP contains all clotting factors found within the plasma, the significant volume, thaw and transport time, slow rate of infusion, and lack of data as an effective therapy all together make it a supportive therapy in the treatment of a major bleeding event due to a new oral anticoagulant.

\section{Recombinant Activated Factor VII (rFVIIa)}

rFVIIa (NovoSeven; Novo Nordisk Inc., Princeton, NJ, USA) is a widely available recombinant antihemophilic clotting factor used off-label in the management of critical bleeding events. It remains questionable if the in vitro effects of rFVIIa will translate to clinical efficacy. Studies on the usefulness of rFVIIa at varying doses to control bleeding events in animal models with dabigatran and rivaroxaban have yielded conflicting results [16, 20]. An in vitro analysis of platelet-poor plasma samples demonstrated the ability of rFVIIa to correct the lag time in thrombin generation, but showed very little effect on the endogenous thrombin potential in both dabigatran- and rivaroxaban-treated patients at doses equivalent to 20,60 , and $120 \mu \mathrm{g} / \mathrm{kg}[21 \bullet$ ]. These findings appear to be confirmed in another in vitro analysis with dabigatran, though suggestive that correction of thrombin generation parameters may be dependent on the concentration of dabigatran [22]. In a recent case report, a patient developed a massive postoperative bleeding event after cardiac surgery due to dabigatran. A cumulative $270 \mu \mathrm{g} / \mathrm{kg}$ dose appeared to slow but was unable to stop the critical bleeding [15]. It is unclear if larger doses of rFVIIa than that used in this case would result in hemostasis.

Very little evidence is available to date evaluating the use of rFVIIa as a reversal agent for apixaban. One in vitro analysis demonstrated the ability of rFVIIa $270 \mu \mathrm{g} / \mathrm{kg}$ to correct the clotting time and thromboelastomeric parameters while having the least effect on the thrombin peak and generation when compared to PCC4 and FEIBA [23]. Given the aforementioned findings and some of the pharmacologic similarities between rivaroxaban and apixaban, it is our hypothesis that rFVIIa would yield similar outcomes to previously published thrombin generation studies and case reports within this class of agents.

The use of rFVIIa to quell a bleeding event due to a new oral anticoagulant remains equivocal. Although it is unclear, the degree and duration of hemostasis may depend on the initial concentration of the anticoagulant. Clinicians treating a patient experiencing a major bleeding event in the setting of a new oral anticoagulant may wish to consider other potential pro-hemostatic agents if available. Use of rFVIIa may be appropriate in patients at risk for bleeding during an urgent procedure when other options are not available.

\section{Prothrombin Complex Concentrates (PCC)}

PCC products are human-derived plasma concentrates. There are three distinct classes of PCC products. PCC4 products contain clotting factors II, VII, IX, and X, while PCC3 products contain clotting factors II, IX, and X, though lack a significant amount of VII. The aPCC products contain clotting factors II, VII, IX, and X, with varying amounts in their activated forms. PCC products are standardized to the factor IX component with varying concentrations of other clotting factors [16]. Certain products may contain other additives such a protein $\mathrm{C}$, protein $\mathrm{S}$, antithrombin, and heparin [24, 25].

\section{PCC3}

Very few data exist with $\mathrm{PCC} 3 \mathrm{~s}$ as a potential reversal agent for the second-generation anticoagulants. There has been no study to date evaluating PCC3s as a potential reversal agent in the setting of dabigatran. In a single case report, approximately $20 \mathrm{U} / \mathrm{kg}$ of PCC3 (Profilnine SD, Grifols Biologicals Inc., Los Angeles, CA, USA) appeared to eliminate the need for further blood transfusions and normalized the aPTT in a patient experiencing hemorrhagic shock from a gastrointestinal bleed while on dabigatran; however, the patient eventually expired of multisystem organ failure [26].

Some clinicians have attempted to 'build' a PCC4 in vivo via administering a PCC3 together with an FVIIcontaining product. Sarode and colleagues administered an estimated $50 \mathrm{U} / \mathrm{kg}$ of Profilnine along with an INRdependent dose of $1.2 \mathrm{mg}$ or $2.4 \mathrm{mg}$ rFVIIa to reverse the anticoagulant effects of warfarin. After measuring incremental levels of FVII and noting $4 \%$ of patients developed an NSTEMI as late as 3 days post administration, the authors recommended using an even lower than studied 
$0.5 \mathrm{mg}$ rFVIIa dose in patients at high risk of thrombosis [9]. No data have been published describing the combined use of PCC 3 and rFVIIa in patients experiencing a bleeding event due to a new oral anticoagulant. It remains unclear what the thrombotic potential would be of this combination given the short half-life of these new oral agents.

Recently, Levi et al. compared the effects of $50 \mathrm{U} / \mathrm{kg}$ PCC3 (Profilnine SD, Grifols Biologicals Inc., Los Angeles, CA, USA) to $50 \mathrm{U} / \mathrm{kg}$ PCC4 (Beriplex, CSL Behring, Hattersheim am Main, Germany) in vivo in patients administered rivaroxaban [27]. While the PCC4 reduced the prothrombin time to a greater extent than the $\mathrm{PCC} 3$, the PCC3 resulted in a larger change in the endogenus thrombin potential (ETP). Neither treatment group experienced a clinically evident thromboembolic event.

\section{PCC4}

Much of the currently available literature using PCC products as reversal agents for the second-generation oral anticoagulants is with PCC4 products. Four studies have evaluated varying laboratory aspects of dabigatran, apixaban, and rivaroxaban after administration of a PCC4 product $[21 \bullet, 23,28 \bullet, 29]$.

It appears that administration of a PCC4 in the setting of therapeutic doses of dabigatran may have no effect on certain common laboratory measures of anticoagulation, but will alter other more sophisticated tests of thrombin generation. The aPTT, ECT, and TT appear to be unaffected after the administration of $25-50 \mathrm{U} / \mathrm{kg}$ PCC4 in the setting of therapeutic levels of dabigatran $[14,28 \cdot]$. The ETP, however, is affected in a dose-dependent manner by PCC4 products, while the lag time to thrombin generation remains unaffected [21•].

Laboratory parameters affected by rivaroxaban and apixaban appear to correct dramatically after administration of PCC4 products. A near normalization of the PT occurs within 15 min post PCC4 infusion and is maintained for $24 \mathrm{~h}$ in subjects given therapeutic doses of rivaroxaban [28.]. The ETP correction appears to be dose dependent, while the lag time to thrombin generation remains unaffected despite escalating doses equivalent to $12.5,25$, and $50 \mathrm{U} / \mathrm{kg}$. The ETP was noted to correct to baseline within $15 \mathrm{~min}$ of administration of $50 \mathrm{U} / \mathrm{kg}$ PCC4 and remain greater than $150 \%$ at $24 \mathrm{~h} \mathrm{[28 \bullet ].} \mathrm{Similarly} \mathrm{with} \mathrm{apixaban,}$ thrombin peak and velocity indexes were corrected with PCC4 $50 \mathrm{U} / \mathrm{kg}$ with slightly less of an effect on the thromboelastometeric parameters when compared with other prohemostatic agents [23].

It is unclear whether the elevated ETP above baseline is necessary for hemostasis, but it also carries increased risk for a thromboembolic event. The lowest effective dose should be used whenever possible, and titration to effect should occur if the situation allows.

\section{FEIBA}

The aPCC, FEIBA, contains clotting factors II, IX, and X mainly in the inactive form and VII in the active form. Studies investigating FEIBA as a potential reversal agent in the setting of the new oral anticoagulants are confined to in vitro or ex vivo analyses and case reports. Small doses of FEIBA, corresponding to $20-40 \mathrm{U} / \mathrm{kg}$, appear to significantly correct the anticoagulant pharmacokinetic and pharmacodynamics parameters of rivaroxaban and dabigatran to near baseline, while larger doses appear to overcorrect the thrombin peak and ETP [21•, 30].

These findings appear to be confirmed in two case reports describing the use of FEIBA in the setting of therapeutic dabigatran. Dager and colleagues described the successful control of a major bleeding event during an atrial fibrillation ablation with the use of FEIBA. During administration of the $26 \mathrm{U} / \mathrm{kg}$ dose, the bleeding appeared to visibly slow within minutes. The bleeding stopped upon the completion of the infusion [14]. Doses smaller than those studied in vitro may be adequate in maintaining sufficient hemostasis during minimally invasive procedures. FEIBA $8 \mathrm{U}$ administered immediately prior to hemodialysis catheter placement was used successfully to control expectant bleeding during the procedure [13]. However, it should be noted that these are case experiences.

There has been no published literature to date describing the use of FEIBA in the setting of a clinical bleeding event due to a direct factor Xa inhibitor. Given the currently available literature, it is reasonable to assume that FEIBA would reverse the anticoagulant effects of rivaroxaban and apixaban based on in vitro and in vivo observations and that their activity is higher up in the coagulation cascade [21•, 23, 28• 29].

\section{Post Intervention Management}

Continuous assessment of the patient needs to occur routinely throughout the hospitalization after the administration of any pro-hemostatic agent. A patient may develop re-bleeding at the initial site of injury or possibly an alternative site. The effects of a concentrated clotting factor or complex concentrate will diminish over a period depending on the half-life of the protein or degree of consumption in clot formation. Moreover, despite obtaining hemostasis, the second-generation oral anticoagulant will remain present within the cardiovascular system for a period dependent on the anticoagulant's initial concentration and half-life, which will be prolonged in selected settings including significant renal dysfunction.

It is also important to consider re-initiation of thromboprophylaxis. Patients are placed on second-generation oral anticoagulants for the treatment and prevention of a thromboembolic event. While the patient may have experienced a 
bleeding event, a continual risk-benefit assessment should take place to prevent a devastating thromboembolic event from occurring. Also, these patients received a potent prohemostatic agent that may continue to drive clot formation well after the effects of the anticoagulant have diminished.

\section{Conclusion}

The second-generation oral anticoagulants, dabigatran, rivaroxaban, and apixaban, have shown an acceptable bleeding risk profile when compared to warfarin. Undoubtedly, the clinician will need to care for a patient at risk for or experiencing a pathological bleeding event in the presence of a second-generation oral anticoagulant. Successful management should include a decision support system that removes potential barriers to necessary interventions, avoids potential delays in therapies, and guides the selection and dosing of the reversal agent.

The initial assessment of the patient is crucial in the treatment of the bleeding patient. Clinical and laboratory monitoring, including continuously assessing the risk of rebleeding and potential for thrombosis, should occur throughout the acute period and continue until discharge from the institution.

Very few data exist to date in the treatment of a pathologic bleeding event due to a second-generation oral anticoagulant. We provide a list of issues including clinical assessment, laboratory parameters, degree of anticoagulation, clotting factors, and complex concentrates that must be considered before initiating treatment. Non-specific prohemostatic agents continue to be investigated as potential options to reversing the anticoagulant effects. There are no robust data to guide the selection and dosing of these prohemostatic agents, leading the clinician to sift and sort through in vitro studies, animal models, and case reports.

\section{Compliance with Ethics Guidelines}

Conflict of Interest A. Josh Roberts, Mark E. Sutter, and William E. Dager declare that they have no conflict of interest.

Human and Animal Rights and Informed Consent This article does not contain any studies with human or animal subjects performed by any of the authors.

\section{References}

Papers of particular interest, published recently, have been highlighted as:

- Of importance

1. Connolly SJ, Ezekowitz MD, Yusuf S, et al. Dabigatran versus warfarin in patients with atrial fibrillation. $\mathrm{N}$ Engl $\mathrm{J}$ Med. 2009;361:1139-51.
2. Patel MR, Mahaffey KW, Garg J, et al. Rivaroxaban versus warfarin in nonvalvular atrial fibrillation. $\mathrm{N}$ Engl $\mathrm{J}$ Med. 2011;365:883-91.

3. Granger CB, Alexander JH, McMurray JJ, et al. Apixaban versus warfarin in patients with atrial fibrillation. $\mathrm{N}$ Engl $\mathrm{J}$ Med. 2011;365:981-92.

4. Healey JS, Eikelboom J, Douketis J, et al. Periprocedural bleeding and thromboembolic events with dabigatran compared with warfarin: results from the randomized evaluation of longterm anticoagulation therapy (RE-LY) randomized trial. Circulation. 2012;126:343-8.

5. Lu G, DeGuzman FR, Hollenbach SJ, et al. A specific antidote for reversal of anticoagulation by direct and indirect inhibitors of coagulation factor Xa. Nat Med. 2013;19:446-51.

6. Thalji N, Patel S, Fruebis J, et al. Reversal of direct factor Xa inhibitors using factor Xa zymogen-like variants. J Thromb Haemost. 2013;11:1-1322 [abstract OC 36.4].

7. Schiele F, van Ryn J, Canada K, et al. A specific antidote for dabigatran: functional and structural characterization. Blood. 2013;121:3554-62.

8. Dager WE. Using prothrombin complex concentrates to rapidly reverse oral anticoagulant effects. Ann Pharmacother. 2011;45:1016-20.

9. Sarode R, Matevosyan K, Bhagat R, et al. Rapid warfarin reversal: a 3-factor prothrombin complex concentrate and recombinant factor VIIa cocktail for intracerebral hemorrhage. J Neurosurg. 2012;116:491-7.

10. Yank V, Tuohy CV, Logan AC, et al. Systematic review: benefits and harms of in-hospital use of recombinant factor VIIa for offlabel indications. Ann Intern Med. 2011;154:529-40.

11. Wójcik C, Schymik ML, Cure EG. Activated prothrombin complex concentrate factor VIII inhibitor bypassing activity (FEIBA) for the reversal of warfarin-induced coagulopathy. Int J Emerg Med. 2009;2:217-25.

12. Kaatz S, Kouides PA, Garcia DA, et al. Guidance on the emergent reversal of oral thrombin and factor Xa inhibitors. Am J Hematol. 2012;87:S141-5.

13. Chang DN, Dager WE, Chin AI. Removal of dabigatran by hemodialysis. Am J Kidney Dis. 2013;61:487-9.

14. Dager WE, Gosselin RC, Roberts AJ. Reversing dabigatran in life-threatening bleeding occurring during cardiac ablation with factor eight inhibitor bypassing activity. Crit Care Med. 2013;41:42-6.

15. Warkentin TE, Margetts P, Connolly SJ, et al. Recombinant factor VIIa (rFVIIa) and hemodialysis to manage massive dabigatran-associated postcardiac surgery bleeding. Blood. 2012;119:2172-4.

16. Kalus JS. Pharmacologic interventions for reversing the effects of oral anticoagulants. Am J Health Syst Pharm. 2013;70:S12-21.

17. Dager WE. Developing a management plan for oral anticoagulant reversal. Am J Health Syst Pharm. 2013;70(10 Suppl 1):S21-31.

18. Tsu LV, Dienes JE, Dager WE. Vitamin K dosing to reverse warfarin based on INR, route of administration, and home warfarin dose in the acute/critical care setting. Ann Pharmacother. 2012;46:1617-26.

19. Van Ryn J, Stangier J, Haertter S, et al. Dabigatran etexilate-a novel, reversible, oral direct thrombin inhibitor: interpretation of coagulation assays and reversal of anticoagulant activity. Thromb Haemost. 2010;103:1116-27.

20. Perzborn E, Gruber A, Tinel H, et al. Reversal of rivaroxaban anticoagulation by haemostatic agents in rats and primates. Thromb Haemost. 2013;110:162-72.

21. - Marlu R, Hodaj E, Paris A, et al. Effect of non-specific reversal agents on anticoagulant activity of dabigatran and rivaroxaban: a randomised crossover ex vivo study in healthy volunteers. Thromb Haemost 2012, 108:217-24. This study evaluated the 
ex vivo effects of various concentrations of FEIBA, rFVIIa, and PCC4 (Kanokad) on the quantitative and kinetic parameters of thrombin after the administration of either dabigatran or rivaroxaban. While PCC4 and rFVIIa affected the investigated thrombin parameters to varying degrees depending on the second-generation anticoagulant studied, FEIBA corrected all parameters regardless of the anticoagulant.

22. Hoffman M, Volovyk Z, Monroe D. Reversal of dabigratran effects by factor VIIa in a cell-based model of coagulation. J Thromb Haemost. 2013;11:1-1322 [abstract OC 36.6].

23. Escolar G, Arellano-Rodrigo E, Reverter J, et al. Coagulation factor concentrates restore alterations in hemostasis induced by a high dose of apixaban: studies in vitro with circulating human blood [abstract2263]. Presented at the 54th ASH Annual Meeting and Exposition, Atlanta, GA, 8-11 Dec, 2012

24. Bershad EM, Suarez JI. Prothrombin complex concentrates for oral anticoagulant therapy-related intracranial hemorrhage: a review of the literature. Neurocrit Care. 2010;12:403-13.

25. Kcentra [prescribing information]. Kankakee, IL: CSL Behring LLC; July, 2013.

26. Dumkow LE, Voss JR, Peters M, Jennings DL. Reversal of dabigatran-induced bleeding with a prothrombin complex concentrate and fresh frozen plasma. Am J Health Syst Pharm. 2012;69:1646-50.

27. Levi M, Moore T, Castillejos CF. Effects of three-factor and fourfactor prothrombin complex concentrates on the pharmacodynamics of rivaroxaban. J Thromb Haemost. 2013;11:1-1322 [abstract OC 36.5].

28. - Eerenberg ES, Kamphuisen PW, Sijpkens MK, et al. Reversal of rivaroxaban and dabigatran by prothrombin complex concentrate: a randomized, placebo-controlled, crossover study in healthy subjects. Circulation 2011, 124:1573-9. This study evaluated the post-administration effect of a PCC4 (Cofact) on certain coagulation laboratory parameters in a small number of patients taking either rivaroxaban or dabigatran. The authors determined that the PCC4 reversed the measured laboratory parameters of rivaroxaban while having no effect on dabigatran.

29. Dinkelaar J, Molenaar PJ, Ninivaggi M, et al. In vitro assessment, using thrombin generation, of the applicability of prothrombin complex concentrate as an antidote for Rivaroxaban. J Thromb Haemost. 2013;11:1111-8.

30. Turecek PL, Schrenk G, Schaedler M. Thrombin generation assay to monitor the reversal of anticoagulants by FEIBA. J Thromb Haemost. 2013;11:1-1322 [abstract PB 4.48-3]. 